\title{
Pathologie de parkings enterrés sous la nappe à Lyon
}

\section{G. SANGLERAT}

Expert honoraire agrée par la Cour de cassation

Professeur honoraire de Mécanique des Sols à l'École centrale de Lyon 182 bis, av, Félix-Faure 69003 Lyon

\section{N. MONGEREAU}

Professeur émérite des universités, URGC Géotechnique, INSA Lyon Expert agréé par la Cour de cassation

41, rue Tronchet 69006 Lyon noel.mongereau@ libertysurf.fr
Quatre parkings, profondément enterrés sous la nappe phréatique, ont été construits à Lyon, à l'abri de parois moulées. Ces dernières ont été réalisées à travers des formations alluviales posées soit sur le granite, soit sur des formations sédimentaires tertiaires. Pour chaque cas, sont exposés les problèmes rencontrés lors de la construction et les surcoûts générés qui reflètent. a posteriori, la prise en compte incorrecte des caractéristiques de chaque site. Les auteurs mettent également en évidence les pièges du radier drainant.

Mots-clés : paroi moulée, piège géologique, renard liquide, renard solide, radier drainant.

\section{Underground parking structures below groundwater in Lyon (France)}

The construction, below groundwater, of new underground parking structures, in the city of Lvon (France), has required to use of diaphragm walls. The diaphragm walls were installed through alluvial deposits and keyed into either granitic or sandstone bedrock. Four case histories of underground parking structure construction are presented. The case histories emphasize the geological, geotechnical, hvdrological challenges encountered, discovered and resolved during construction.

Key words : diaphragm walls, geologic pitfall, liquid and solid piping, drainage blanket. 


\section{Contexte géologique lyonnais}

\section{1}

\section{Presqu'île lyonnaise}

Pour le secteur situé dans la presqu'île de Lyon, limité à l'est par le Rhône et à l'ouest par la Saône, les terrains rencontrés en profondeur sont les mêmes que ceux trouvés sous les collines de la Croix-Rousse et de Fourvière. On rencontre, de bas en haut :

- socle cristallin

- terrains tertiaires ;

- formations quaternaires.

\section{T.1.1.}

\section{Socle cristallin}

Le socle cristallin comporte deux grands ensembles : - les roches métamorphiques, type gneiss ;

- les roches plutoniques, type granite.

Au cours de leur histoire, ces terrains sont restés pendant un temps très long à l'atmosphère et ont subi des phénomènes d'altération. A leur surface il peut subsister soit des fragments sous forme de blocs emballés souvent dans une matrice argileuse, résidu d'altération du socle ou apport extérieur, soit des zones altérées de moindre résistance qui font suite latéralement à des zones plus cohérentes.

\section{1 .8}

\section{Terrains tertiaires}

Le matériau le plus connu, parce que le plus important en volume déposé, correspond à des sables siliceux, calcaires et micacés, jaune clair ou gris, contenant des minéraux de forte densité dits minéraux « lourds » - densité supérieure à 2,9- (épidote, grenat, etc.). Ces derniers minéraux permettent une bonne identification du sédiment. Dans sa masse, ce sédiment peut comporter des niveaux consolidés en grès (niveaux de forme et de dimension variables). Fondamentalement, le terme de molasse est réservé à ces niveaux consolidés. On a ensuite étendu l'appellation molasse à l'ensemble du sédiment.

Il convient cependant de noter que, latéralement, on peut passer à des formations argileuses en l'espace de quelques mètres. Cela peut avoir des conséquences importantes lorsqu'on décide d'effectuer des pompages soit pour rabattre la nappe, soit pour alimenter des réseaux de distribution, soit pour faire fonctionner des pompes à chaleur.

A la base du Tertiaire, existe un faciès allant du conglomérat (diamètre des éléments : $0,40 \mathrm{~m}$ ) au sable fin. Les éléments sont ceux de toutes les roches du Massif central, y compris les calcaires du Lias. Le ciment est argilo-ferrugineux et la couleur est rouge ou verte. Confondu à tort avec des arènes granitiques, ce faciès est partie intégrante des terrains tertiaires.

\section{Terrains quaternaires}

Sous le nom de " moraine $)$, on range tous les terrains alluviaux liés directement aux glaciers würmiens, tandis qu'on réserve celui de « fluvio-glaciaire » pour les alluvions où l'intervention des eaux courantes de fusion a été prépondérante.

Les formations fluviatiles les plus récentes sont appelées alluvions.

\section{2}

\section{Plaine de Lyon}

La plaine de Lyon, à l'est du Rhône, est constituée, sous environ $20 \mathrm{~m}$ d'alluvions, de formations tertiaires présentes sous forme de molasse sableuse et gréseuse, passant latéralement à des formations argileuses. On ne connait pas le niveau de base de cette formation.

Le sondage le plus profond réalisé (Sanglerat et al., 1999) a traversé, sous 21 m d'alluvions, la molasse sur 24 m d'épaisseur, sans atteindre la base de cette formation. Ce sondage a été effectué en 1994 par le pénétromètre statique dynamique AMAP'sols, sur le site du parking Foch cité ci-dessous.

L'épaisseur de la molasse est certainement très grande. Un forage réalisé près de Saint-Laurent-deMure (à $20 \mathrm{~km}$ à l'est de Lyon) a mis en évidence une épaisseur de $340 \mathrm{~m}$ (Mongereau, 2001).

\section{Hydrogéologie}

Au plan hydrogéologique, l'eau est présente dans l'ensemble des formations décrites ci-dessus :

- entre Saône et Rhône, auxquels la nappe se raccorde, le contexte aquifère est simple, l'eau ayant des caractéristiques chimiques voisines de celles des rivières bordières :

- dans la plaine de Lyon, tout en se raccordant au Rhône, la nappe est suralimentée par les eaux artésiennes et profondes de la molasse, aux caractéristiques incrustantes prononcées.

Dans les deux cas, la nappe se situe vers $6 \mathrm{~m}$ de profondeur.

Les eaux de la molasse ont donné lieu à de nombreuses datations par le C14. Leur âge varie entre 2800 et 4500 ans. Le C14 constitue alors un excellent outil d'identification des eaux en provenance du réservoir molassique (identification initiale, suivi dans le temps des débits, identification de fuites).

Le lecteur qui souhaiterait approfondir les données géologiques et hydrogéologiques résumées ci-dessus pourra se reporter à la bibliographie citée par N. Mongereau (2001), ainsi qu'à la synthèse géologique du Sud-Est de la France (1984). 


\section{Parking souterrain de la Bourse (1990)}

Pour ce parking de sept niveaux, d'une surface de $1922 \mathrm{~m}^{2}$, on a réalisé une paroi moulée d'une hauteur variant de 52 à 57 mètres, ancrée dans le granite (marché de 43709506 francs notifié le 30 novembre 1990). Les reconnaissances géotechniques initiales avaient montré, entre 21 et $33 \mathrm{~m}$ de profondeur, la présence localisée de blocs. Ceux-ci ont alors été considérés comme amas de blocs isolés au sein des alluvions. Hélas, ils ont été rencontrés sur toute l'emprise de la paroi qui a été réalisée à l'hydrofraise, cette méthode permettant de réduire les hors profils au droit de la couche continue de blocs. En raison de l'accroissement des difficultés de forage, l'entreprise a introduit une réclamation s'élevant à 12782382 francs TTC, correspondant * au coût supplémentaire de travaux non prévus dans le marché forfaitaire, engendré par des sujétions imprévues rencontrées dans le sous-sol n). En raison du retard de 25 jours survenu, le maître d'ouvrage voulait appliquer des pénalités s'élevant à 762669 francs.

Les reconnaissances initiales ont comporté deux sondages destructifs avec essais pressiométriques (de $30 \mathrm{~m}$ de profondeur) et deux sondages carottés dont un seul foré jusque dans le granite, à $56,20 \mathrm{~m}$ de profondeur. Les informations fournies par ce dernier n'ont pas été analysées correctement et, de ce fait, il n'était pas possible d'en tirer les conclusions utiles.

L'analyse faite a posteriori de ce sondage montre que, sous-jacent à l'accumulation de blocs trouvée entre 21 et $33 \mathrm{~m}$ de profondeur, on rencontre, à partir de 35,50 m, jusqu'à $48,60 \mathrm{~m}$, des niveaux de sables argileux qui correspondent aux formations « compréhensives $x^{3}$, comme l'on dit en géologie, de la molasse sableuse.

Ces niveaux ont été considérés initialement comme des alluvions fluviatiles quaternaires sablo-argileuses. En conséquence, la présence de blocs pouvait ètre considérée comme ponctuelle et ne pas provoquer de complications pour le forage des parois moulées. Mais, en fait, l'appartenance de ces niveaux au Tertiaire, qui ne peut échapper à un géologue connaissant bien la région, doit naturellement inciter à penser que, lors de la fonte des glaciers, s'est produit un dépôt de blocs pratiquement continu sur une grande surface, d'où un risque de difficultés permanentes de forage des parois. C'est bien ce qui s'est produit et a conduit à la réclamation formulée par l'entreprise.

La présence d'une telle accumulation de blocs ne peut en aucun cas être considérée comme un aléa géologique, c'est-à-dire un fait lié au hasard, mais constitue au contraire un piège géologique $e^{2}$ non détecté par manque et d'une interprétation géologique rattachée au contexte local et d'une reconnaissance adaptée (un plus grand nombre de sondages aurait été nécessaire).

\footnotetext{
${ }^{1}$ « Compréhensive $»$ signifie, selon l'usage des géologues, contemporaine mais de nature différente.

${ }^{2}$ Un piège géologique est une zone d'extension géographique variable où les terrains n'ont pas la nature ou la disposition à laquelle on s'attendait, ce qui forcément induit des contraintes techniques.
}

Il est possible de plus que la limite supérieure des formations tertiaires - ici le substratum des alluvions présente des points bas, zones où préférentiellement l'accumulation des blocs peut être plus importante, d'où accroissement des difficultés de mise en œuvre des parois.

Par jugement $n^{\circ}$ 9502417, rendu le 1er décembre 1999, la $3^{e}$ chambre du Tribunal Administratif de Lyon a:

- condamné le maitre d'ouvrage à payer à l'entreprise, au titre des sujétions imprévues, le tiers de 12065958 francs (réclamation initiale moins abattement), soit 4021999 francs ;

- déclaré les pénalités de retard injustifiées.

Les deux parties ont fait appel.

\section{3}

\section{Parking République (1990)}

Ce parking enterré de 887 places sur sept niveaux a nécessité la réalisation d'une paroi de $0,80 \mathrm{~m}$ d'épaisseur, composée de 87 panneaux de hauteur variant entre 23 et $26 \mathrm{~m}$, selon une trame de $4,60 \mathrm{~m}$ de large, avec des raccords entre panneaux par tubes joints, la base de ces panneaux devant être encastrée dans le granite (marché forfaitaire approuvé le 13 décembre 1990).

Les reconnaissances préliminaires ont comporté 6 sondages carottés avec essais pressiométriques sur une profondeur de $30 \mathrm{~m} ; 9$ sondages avec enregistrement de paramètres ont ensuite été réalisés par l'entreprise avant le début des travaux ; 4 sondages carottés ont été réalisés après sinistre (SC 91-92, SS 21-22).

Pour améliorer l'étanchéité du raccordement pied de paroi au substratum résistant, il a été réalisé deux types d'injection:

- injection en pied par une jupe de $6 \mathrm{~m}$ de haut par 4 forages sur la largeur de 4,60 m de chaque panneau: ces injections verticales ayant donc un entraxe de $1,15 \mathrm{~m}$;

- bouchon injecté de bentonite ciment en arrière et à la base de la paroi sur une hauteur de $4 \mathrm{~m}$ avec 2 injections en quinconce de $3 \mathrm{~m}$ d'entraxe ; la base de ce bouchon injecté atteint $1 \mathrm{~m}$ sous le pied de la paroi.

Ce dispositif devant permettre, en cours de travaux, la détection d'anomalies en pied de paroi dont l'étendue en plan dépasserait $1,15 \mathrm{~m}$ à $1,50 \mathrm{~m}$ selon la zone.

Le 2 septembre 1992, à 8 heures 30, un débourrage important $\left(200 \mathrm{~m}^{3}\right)$ s'est produit au niveau du $7^{\circ}$ soussol, au droit des panneaux 79 et 80 qui constituaient la limite de la zone excavée la veille dans le cadre du déroulement normal du terrassement, la paroi étant découverte côté intérieur depuis 24 heures environ.

Simultanément, un affaissement d'environ $4 \mathrm{~m}$ survenait dans la cave du magasin Tandy située à proximité de la paroi moulée, selon un fontis d'allure elliptique au droit du panneau 79 (Fig. 1). Il y avait risque important d'effondrement des immeubles anciens en maçonnerie de pierre longeant le parking. 


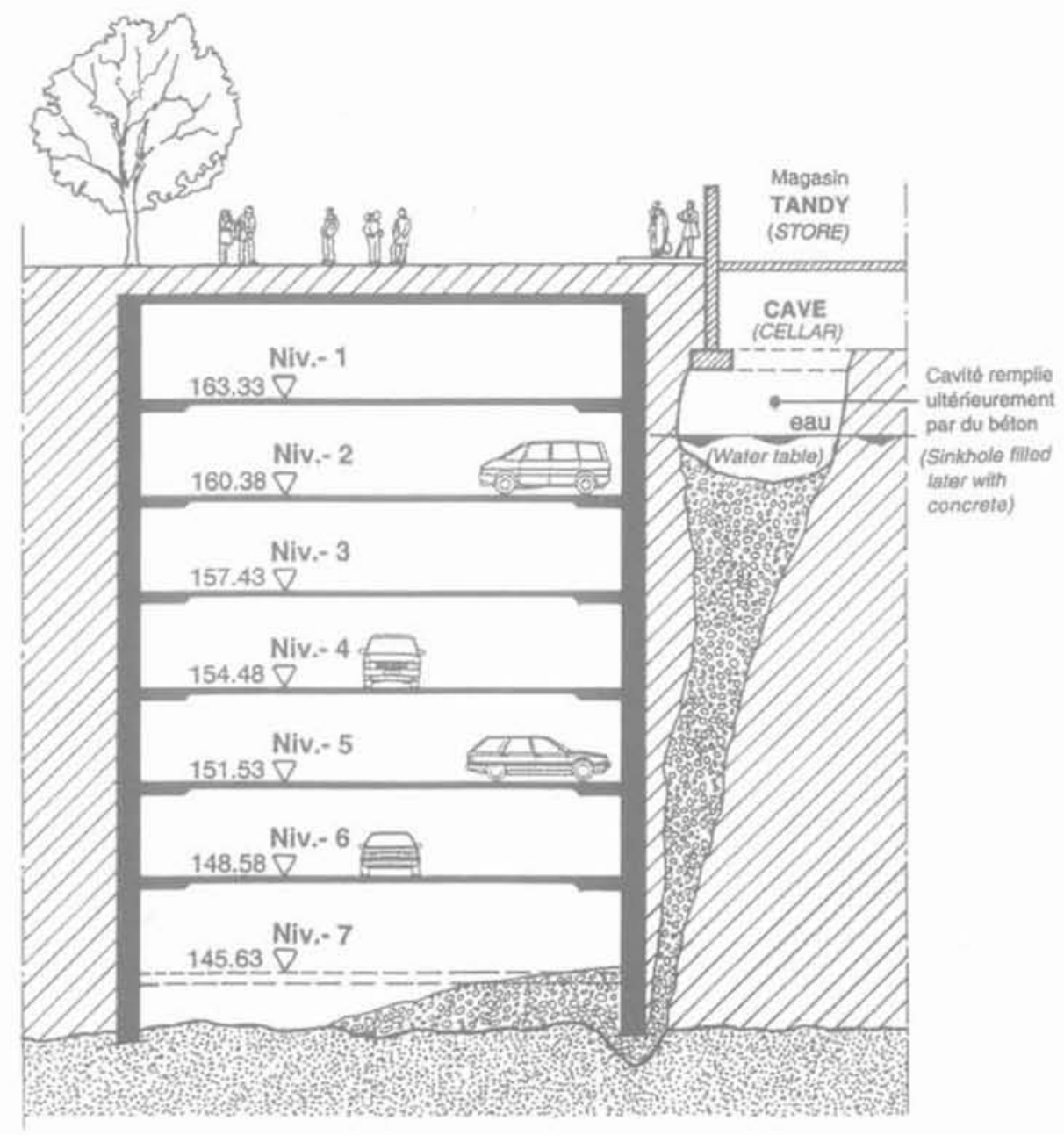

FG. 1 Renard solide et fontis (parking République).

Solid piping and swallow hole.

Ce sinistre a fait naturellement l'objet d'un constat d'urgence ordonné par le Tribunal de Grande Instance, puis d'une expertise devant le Tribunal Administratif de Lyon.

Les experts judiciaires ont montré que :

- la géologie côté Rhône, à l'est de la ligne SC2-SC92 (Fig. 2), est différente de celle des autres parties du parking, pour lesquelles le faciès du granite est relativement sain et le toit du substratum a une allure pratiquement plane :

- le sondage SS22 ayant détecté une anomalie caractéristique très profonde, il y a donc une très forte présomption d'incidence géologique localisée, thalweg accusé, largeur réduite voire dissymétrique (Fig. 2) ;

- dans la zone du sinistre, toutes les parois n'ont pu atteindre le rocher sain, un décalage important $(90 \mathrm{~cm})$ existe entre les bases des panneaux 79 et 80 (Fig. 3) ; malgré les forages complémentaires verticaux sous parois, accompagnés d'injections, il a pu subsister un vide de l'ordre de 30 à $40 \mathrm{~cm}$ permettant le passage d'un renard solide ${ }^{3}$ à travers les zones de gneiss broyé ou fracturé ;

S Un renard solide est un renard (tiquide) accompagné d'un volume très important de terrain.
- il n'est pas possible d'exclure un défaut d'exécution en pied de paroi.

Le préjudice allégué par l'entreprise s'élevait à 7 millions de francs hors taxes.

Le rapport d'expertise judiciaire, déposé en février 1995, proposait d'imputer le coût des travaux de réfection, ainsi que les conséquences des désordres, comme suit :

- entreprise : $2 / 3$

“ défauts possibles d'exécution de la base de la paroi et de ses organes d'étanchéité )) :

-maître d'ouvrage : $1 / 3$

“accident géologique probable ».

Après le dépôt de ce rapport, les parties ont dû se rapprocher car il n'y a pas eu de suites judiciaires.

\section{4}

\section{Parking de la Cité judiciaire (1991)}

La construction du nouveau palais de justice de Lyon, sur une surface au sol de $7000 \mathrm{~m}^{2}$, a été précédée par l'exécution de quatorze sondages de $28 \mathrm{~m}$ de profondeur pour essais divers (granulométrie, Lefranc...) 


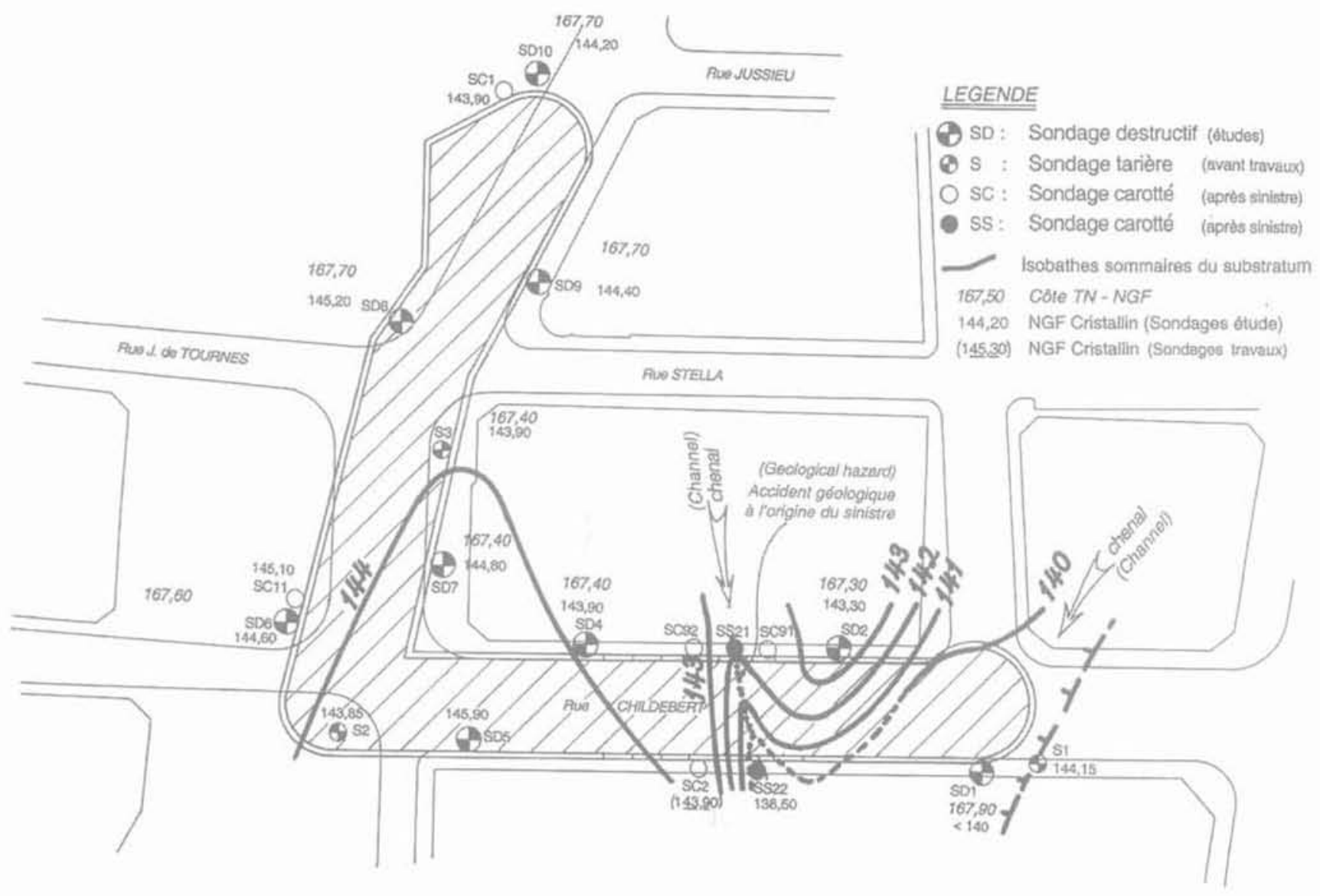

FG.2 Implantation des sondages (parking République). Boreholes draft.

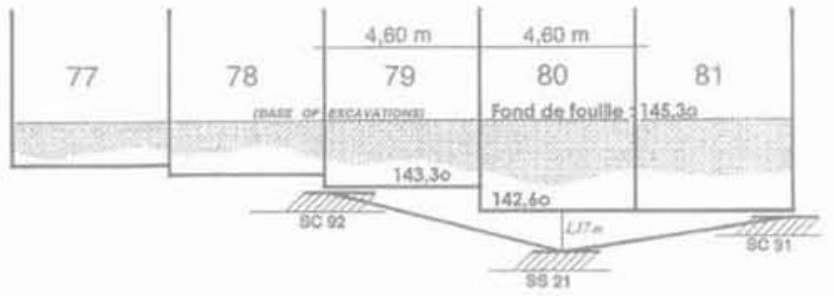

FG. 3 Profil du granite sain au droit de la zone de renard.

Outline of granite near the solid piping.

et d'un seul sondage carotté également de $28 \mathrm{~m}$ avec prélèvement d'échantillons intacts de molasse. Un sous-sol enterré de six niveaux, à usage de parkings (publics et privés), s'étend sous toute la surface du bâtiment, sur une profondeur de $20 \mathrm{~m}$. Plutôt que de prevoir un cuvelage étanche, qui aurait été parfaitement possible, le maître d'cuvre a prescrit un radier drainant, ce qui était, à l'époque, une première à Lyon dans ce type de sol.

La réalisation de la fouille du parking, à l'intérieur d'une enceinte en paroi moulée épaisse de $0,80 \mathrm{~m}$ et profonde de $29 \mathrm{~m}$ (marché forfaitaire notifié le 19 juillet 1991), a rencontré de grandes difficultés (perturbant gravement le déroulement du chantier) résultant principalement de deux éléments :
- erreur sur le niveau du toit de la molasse. D'après les sondages, le toit de la molasse devait se trouver entre 147,39 et 148,98 NGF ; en réalité, le toit de la molasse mémorisée ${ }^{4}$ sur la paroi varie de 147,51 à 150,30 avec une valeur moyenne de 149,29 ; on peut donc considérer que la différence moyenne entre les prévisions faites avant travaux et les constatations après terrassements est de $1,36 \mathrm{~m}$ le long du périmètre de la fouille ; le toit de la molasse a donc été trouvé beaucoup plus haut que prévu :

- erreur sur la perméabilité de la molasse. Estimée par un nombre insuffisant d'essais Lefranc à $3 \times 10^{-5} \mathrm{~m} / \mathrm{s}$, les perméabilités observées se sont montrées bien inférieures, de l'ordre de $10^{-5}$ à $10^{-7} \mathrm{~m} / \mathrm{s}$, d'où des difficultés pour rabattre la nappe par le système de pompage prévu (rayon d'action des puits insuffisant).

A ces deux éléments très défavorables, il convient d'en ajouter quatre :

- le caractère incrustant de l'eau qui a entrainé de nombreux problèmes pour la phase travaux (colmatage des puits de pompage, des pompes et des puits de rejet). Souvent, pour faire face aux désordres provoqués par le carbonate de calcium, certaines entreprises n'hésitent pas à ( balancer ») de l'acide chlorhydrique ; cette

\footnotetext{
4 Sur une parol dégagée lors du creusement de la fouille, le niveau du toit de la molasse apparait nettement ; il correspond au changement d'aspect de la surface du béton : granuleux dans les alluvions, lisse dans la molasse.
} 
méthode brutale nous parait peu écologique ; fort heureusement, elle n'a pas été utilisée dans le cas du parking de la Cité judiciaire ;

- la présence des particules les plus fines de la molasse (particules colloïdales) dont on n'avait pas prévu la floculation (colmatage des puits et pompes) :

- l'artésianisme de la nappe générant des débits considérables et conduisant, en cours de creusement sous le niveau piézométrique, à l'entrainement sur le fond de fouille des particules sableuses de la molasse, provoquant ainsi de petits renards solides ascendants ; ces derniers ont créé des vides, quelquefois importants, sous certaines semelles de fondations des piliers centraux. L'artésianisme de la nappe de la molasse est de l'ordre de $2 \mathrm{~m}$ dans cette zone de Lyon (Mongereau, 2001):

- l'origine de l'eau provenant du réservoir molassique générant des débits considérables $\left(350 \mathrm{~m}^{3} / \mathrm{h}\right)$, tant pendant la phase travaux qu'ultérieurement ad vitam aeternam compte tenu de la réalisation d'un radier drainant.

Ces six éléments défavorables, mal maîtrisés pendant la phase travaux, ont conduit à des modifications dans la conduite des techniques de mise hors d'eau utilisées et à une refonte complète et de la conception et de la réalisation du radier drainant. En particulier, les 180 pointes filtrantes prévues au marché initial ont dû être abandonnées car leur rayon d'action était trop faible, puisque la perméabilité de la molasse avait été surestimée. Elles ont èté remplacées par des tranchées drainantes et des puits.

Le délai initialement prévu pour les travaux était de 34 mois et a été porté contractuellement à 40 mois 8 jours. Finalement, le retard effectif dans la mise en service du parking a été de 78 jours.

Les préjudices allégués par les parties s'élevaient à 46 millions de francs hors taxes, se décomposant comme suit :

- réclamation des deux entreprises : 25,5 millions de francs ;

- préjudices allégués par le maître d'œuvre ; 5,8 millions de francs ;

- préjudices allégués par les deux maitres d’ouvrage 14,7 millions de francs.

Les experts judiciaires ont déposé auprès du Tribunal Administratif de Lyon deux rapports techniques le 3 juin 1995 et le 25 juillet 1997, puis, le 7 mars 2000, un troisième rapport analysant les préjudices consécutifs au retard dans la livraison des parkings.

Dans leurs rapports techniques, les experts ont proposé les imputations suivantes :

- conception : $70 \%$, dont $50 \%$ à la charge de la maitrise d'œuvre et $20 \%$ à la charge des entreprises ; - exécution : $30 \%$ à la charge des entreprises.

Par jugement du 4 juillet 2002, le Tribunal Administratif de Lyon:

- a débouté les maîtres d'ouvrage et le maître d'œuvre, car les travaux avaient été réceptionnés sans réserve;

- a accordé à l'une des entreprises 9,2 millions de francs, assortis des intérêts moratoires.

Ce jugement n'a fait l'objet d'aucun appel.

\section{Remarques concernant les radiers drainants}

Le parking de la gare de la Part-Dieu, construit à partir de 1994, conçu comme celui de la Cité judiciaire, a fort heureusement bénéficié des erreurs commises lors de la réalisation de ce dernier. Situés géologiquement et hydrogéologiquement dans les mêmes conditions, les débits extraits puis restitués en milieu naturel sont de $1000 \mathrm{~m}^{3} / \mathrm{h}$.

Le pompage pour ces deux parkings correspond à l'extraction, tous les ans, de $11826000 \mathrm{~m}^{3}$ d'eau de très bonne qualité, ce qui représente sensiblement neuf fois la production annuelle d'eau d'Évian, de caractéristiques chimiques semblables - le réservoir géologique est le même. Le paradoxe est qu'aucune utilisation n'a été prévue pour les eaux de pompage provenant de ces deux parkings, qui sont rejetées à leur périphérie. Elles risquent alors d'envahir à plus ou moins long terme les parkings et sous-sols voisins moins profonds. En outre, les eaux de pompage, en se mélangeant aux eaux de surface, se chargent inévitablement en éléments bactériologiques et chimiques polluants.

La presse (Le Figaro Lyon du 11 septembre 2001) s'est fait l'écho de cette aberration qui s'ajoute aux conséquences des constructions anarchiques en sous-sol et à l'exploitation incontrôlée de la nappe superficielle et profonde, notamment par les pompes à chaleur qui conduisent à une élévation de la température de la nappe de plusieurs degrés dans certains quartiers. Aussi, comme nous l'avons à plusieurs reprises suggéré, les responsables de la Communauté urbaine de Lyon ont décidé une reprise du suivi de la nappe et s'orientent vers la mise en cuvre d'un Plan d'occupation du sous-sol.

Par ailleurs, la décision de réaliser un radier drainant peut paraître quelquefois conduire à une économie dans le coût de construction. Mais cela implique inévitablement des frais d'exploitation importants ad vitam aeternam. Il y a donc transfert d'une économie initiale (quelquefois illusoire) au détriment du futur.

Si la technique du radier drainant est admissible dans le cas où les débits à pomper sont très faibles (quelques mètres cubes par heure), par contre, à notre avis, cette solution doit être proscrite dans le cas de débits très importants, comme ceux mentionnés ci-dessus.

\section{5}

\section{Parking Foch (1994)}

Le parking Foch, situé 55, avenue Foch, Lyon 6e, a été créé dans le cadre de la rénovation d'un immeuble. Les travaux ont notamment comporté la réalisation d'une enceinte étanche de $22,50 \mathrm{~m}$ sur $33,50 \mathrm{~m}$, pour la création de 220 places de parking en sous-sol, du niveau -2 au niveau -8 . Les alluvions d'une épaisseur de 25,80 m reposent sur la molasse. La profondeur de la fouille a été de $22,50 \mathrm{~m}$. La paroi moulée, d'épaisseur $0,80 \mathrm{~m}$, a été réalisée d'août 1994 à décembre 1994. Son arase est à $3,20 \mathrm{~m}$ et sa base à $31 \mathrm{~m}$ de profondeur.

Le 7 novembre 1994, lors de la réalisation du panneau 30D, l'excavatrice a eu une avarie de chantier. La creuse a été arrêtée à $-27 \mathrm{~m}$, soit $4 \mathrm{~m}$ au-dessus de la cote prévue et entièrement dans la molasse, et le panneau a été bétonné en l'état.

Pour tenter de reconstituer la fiche initialement prévue, il a été décidé d'effectuer des injections dans la molasse. Or, vouloir injecter de la molasse lyonnaise est une vue de l'esprit !

Le 16 août 1995, lorsque le terrassement a atteint le plancher bas du $7^{\text {e }}$ sous-sol, s'est produit un renard important à la base du panneau 30D, générant en surface un fontis évalué à $100 \mathrm{~m}^{3}$, mettant gravement en 
danger la stabilité d'un immeuble voisin et permettant une communication directe entre la nappe et la fouille qui a été inondée sous $16,20 \mathrm{~m}$ d'eau. Afin de colmater la « fenêtre ») ainsi créée à la base du panneau $30 \mathrm{D}$, un traitement par jet-grouting a été effectué. Les travaux de pompage ont repris à l'intérieur de la fouille et un deuxième renard s'est produit le 6 février 1996 au même endroit.

Finalement, l'étanchéité provisoire de la fouille a été obtenue grâce à un traitement par congélation et injection, ce qui a permis la réception du chantier le 6 janvier 1998. Le retard sur le planning a été de 21 mois et les préjudices allégués par les différentes parties s'élèvent à 42 millions de francs répartis comme suit :

- maître d'ouvrage : 4,5 millions de francs ;

- entreprises : 37,5 millions de francs.

Deux rapports ont été déposés au Tribunal de Grande Instance en novembre 1999, un troisième en mai 2002. En janvier 2003, aucun jugement n'a été prononcé.

\section{6}

\section{Conclusion}

Le cas du parking Foch est un exemple caractéristique d'une faute d'exécution (défaut de fiche d'une paroi), suivie d'erreurs dans l'exécution des méthodes adoptées pour remédier aux insuffisances et désordres observés. Les trois autres exemples illustrent les difficultés d'adaptation aux sites dans lesquels s'intègrent les parois moulées lorsque la géologie est complexe et que les sondages prescrits initialement sont insuffisants en nombre et en profondeur.

Ces difficultés résultent du manque d'analyse géologique et géotechnique ponctuelle du site et de l'absence d'analyse globale liée à la géologie locale. Elles mettent en évidence l'existence des pièges géologiques qui ne peuvent être surmontés que si l'on a mis en œuvre tous les moyens dont on dispose pour les détecter en temps utile et que l'on possède une connaissance très fine de la géologie locale.

Les erreurs commises à différents stades ont provoqué des surcoûts très importants (de 1 à 7 millions d'euros), et quelquefois des retards considérables qui ont conduit inévitablement les intervenants devant les tribunaux. Près de dix ans après les désordres, trois des quatre litiges ne sont pas encore arrivés à leur terme. Que de temps et d'argent perdus !

Il n'est pas sans intérêt de remarquer que les travaux ont èté réalisés par différentes entreprises renommées, sous le couvert de bureaux de contrôle différents mais, hélas, pour aucun des quatre parkings, il n'avait été prévu de a mission de maitrise d'œuvre géotechnique 》) (missions types G.2, G.3 et G.4), ce qui est fort regrettable.

Ajoutons enfin que la technique du radier drainant, qui a pu séduire certains, n'est pas toujours la meilleure solution ni au point de vue économique général global (coût d'investissement + coût d'exploitation à long terme), ni au point de vue écologique, particulièrement lorsque les débits pompés sont élevés.

\section{Bibliographie}

BRGM - Synthèse géologique du Sud-Est de la France. Mémoire n ${ }^{\circ} 125$ et atias $n^{\circ} 126,1984$.

Mongereau N. - Géologie de Lyon. ELAH,
Lyon, 2001, 95 pages, 20 photographies, 1 tableau, 21 figures.

Sanglerat G.. et al. - « Pénétration statique AMAP'sols dans les graviers sableux denses, la molasse et les marnes compactes $x$. Revue française de géotechnique, $n^{\circ} 87,1999$, p. 43-54. 